\title{
Signos, legibilidad y diagnóstico: El problema del dolor en la infancia, 1870-1920
}

\author{
Leticia FERNÁNDEZ-FONTECHA RUMEU \\ University of Greenwich \\ leticiafontecha@gmail.com
}

Recibido: $13 / 06 / 2014$

Aceptado: 02/07/2014

\section{RESUMEN}

El presente artículo investiga la controversia sobre el dolor infantil a finales del siglo XIX y principios del XX desde la perspectiva de la historia de las emociones, explorando cómo la expresión emocional del sufrimiento infantil, representado de forma paradigmática por el llanto y el grito, fue interpretado de diferentes maneras por las diferentes figuras profesionales investidas de una autoridad performativa sobre dichas manifestaciones. Al comparar discursos de científicos y psicólogos con discursos pediátricos, el presente artículo sugiere que mientras los primeros defendían la práctica insensibilidad al dolor durante la infancia y una menor sensibilidad en edad infantil que en edad adulta, los pediatras emplearon el dolor como una vía para diagnosticar las dolencias infantiles.

Palabras clave: Historia de la infancia, dolor infantil, darwinismo, fisiología, psicología experimental, pediatría, diagnóstico.

\section{Signs, legibility and diagnosis: The problem of pain in childhood, 1870-1920}

\begin{abstract}
This paper visits the disputed place of children pain between the late $19^{\text {th }}$ Century and the early $20^{\text {th }}$ Century from the perspective of the History of Emotions. It explores how the emotional expression of children suffering, chiefly represented by cries and screams, was subject to different interpretations depending on the different professional bodies invested with the performative authority required to shape its meaning. A comparison between the discourse of scientists and psychologists and that of pediatricians allows to infer how, whereas the former claimed children were essentially insensitive to pain and had in general a lower vulnerability to pain than adults, pediatricians used pain as a path to diagnose children sickness.
\end{abstract}

Key words: History of childhood, infant pain, Darwinism, physiology, experimental psychology, paediatrics, diagnosis.

Sumario: Introducción. 1. La negación del dolor. 2. El lenguaje del dolor 3. Conclusiones. 4. Referencias bibliográficas. 


\section{Introducción}

A algunos les bastará la mención de las palabras "niños" y "dolor" para evocar las fotografías de niños llorando tomadas por Oscar Rejlander, empleadas por Charles Darwin (1809-1882) en su obra La expresión de las emociones en el hombre y en los animales. Una de ellas llevaba en el libro el título de "aflicción moral" (Mental Distress), aunque fue más conocida como Ginx's Baby, y tuvo tanto éxito que Rejlander llegaría a vender 250.000 tarjetas de visita con ese motivo. ${ }^{1} \mathrm{El}$ fotógrafo representa lo que, según Darwin, es la fisionomía característica de un infante "que gimotea o llora": los ojos cerrados, "de manera que la piel a su alrededor está arrugada, y la frente fruncida", la boca abierta y los labios "retraídos de una manera peculiar" y "enseñando más o menos" los dientes. Darwin creía que los "gritos violentos y prolongados" de los niños eran expresiones emocionales asociadas con "el dolor, el hambre moderada o la desazón". ${ }^{2}$ Pero entonces, ¿cómo saber por qué llora este niño en concreto? ¿Será Ginx's Baby una representación de dolor, hambre, frustración o de aflicción moral, como indicaba Darwin en su libro? ¿Contiene acaso esta imagen información valiosa desde el punto de vista médico? En el contexto médico de finales del siglo XIX y principios del siglo XX, la respuesta habría sido rotundamente afirmativa. Al explorar la interpretación del grito y el llanto infantiles en el cambio de siglo se desvela el debate de la época que es objeto de este artículo: ¿son capaces los niños, no ya de expresar, sino de sentir dolor?

El infante dolorido, en tanto que sujeto de investigación, reúne los campos hasta ahora disjuntos de la historia de la infancia y la historia de la medicina. Habida cuenta de que son poquísimos los campos de estudio que han conocido un crecimiento tan efervescente como la historia de la infancia, con tantas áreas de especialidad que ha llegado a ser descrita como una "masa de hebras enredadas" ${ }^{3}$, es sorprendente que el dolor infantil haya recibido una atención tan escasa por parte de los historiadores de la infancia y de la medicina, aunque un examen detenido permite detectar ciertos factores que favorecen o explican esta deficiencia. ${ }^{4}$ Las obras publicadas que repasan la historia del dolor infantil, escritas en su mayoría por profesionales del campo de la psicología, aducen que las creencias sobre la sensibilidad de niños e infantes experimentaron grandes cambios durante este periodo, llegando a la conclusión de que "el escepticismo hacia el dolor infantil caracterizó gran parte de la investigación y las prácticas clínicas durante el siglo XX" ${ }^{5}$ De manera semejante, la historiadora Joanna Bourke, tras haber examinado recientemente la historia del dolor infantil en su libro

1 SMITH, Jonathan: Charles Darwin and Victorian Culture, Cambridge: Cambridge University Press, 2006, p 226.

2 DARWIN, Charles: The Expression of Emotions in Man and Animals, London, John Murray, 1872, p. 147. La traducción es mía, como en el resto del texto, a menos que se indique lo contrario.

3 COOTER, Roger: "Introduction", en Roger Cooter (ed.):In the Name of the Child: Health and Welfare, 1880-1940, London, Routledge, 1992, p. 1.

4 Elissa N. Rodkey y Rebecca Pillai Riddell dedicaron un artículo a este objeto en 2013 y llegaron a la conclusión de que, pese a la importancia del registro histórico para el estudio de los presupuestos médicos sobre la experiencia infantil del dolor, relativamente pocas investigaciones habían repasado esta historia.

5 RODKEY, Elissa y PILLAI RIDDELL, Rebecca: "The Infancy of Infant Pain Research: The Experimental Origins of Infant Pain Denial”, The Journal of Pain, 14, (2013), p. 338. 
The Story of Pain, ha llamado la atención sobre un proceso histórico que llevó a la revisión de la consideración del dolor infantil, haciendo que "muchos científicos y médicos defendieran que los infantes eran casi totalmente insensibles al dolor". ${ }^{6}$ Aunque esta narrativa sirve para describir, al menos en parte, la literatura sobre el dolor infantil del siglo XIX y de inicios del siglo XX, me gustaría desarrollar esta línea de argumentación para extenderla al campo de la historia de la pediatría. De hecho, el trabajo de pediatras como Charles West y Richard Tonson Evanson puede servir para refutar las suposiciones de que los pediatras albergaban "nociones igualmente ingenuas acerca del sufrimiento en la infancia"7.

El argumento central de este artículo consiste en señalar cómo, pese a que muchos científicos proclamaran la práctica insensibilidad de los neonatos al dolor y una sensibilidad menor en niños que en adultos, entendiendo el llanto como un acto reflejo, los pediatras estaban empleando el dolor y sus signos expresivos como una herramienta para diagnosticar patologías infantiles tanto en la teoría (manuales) como en la práctica (casos clínicos). Así pues, este artículo aspira a poner en duda la idea de que las emociones se corresponden de forma mecánica e invariable con ciertos gestos corporales. ${ }^{8}$ Para ello, muestra cómo el grito y el llanto crearon "cuerpos emocionales" distintos en el contexto pediátrico y en los laboratorios. Para arrojar luz sobre esta problemática, es importante investigar cuáles eran para los fisiólogos, los psicólogos, los médicos y los pediatras las causas del dolor en los niños o, en su caso, los motivos por los cuales este dolor no existía.

Este estudio da por buena la existencia de políticas del dolor, una idea defendida por por varios autores cuyas aportaciones han sido relevantes para la historia del dolor ${ }^{9}$. Cada vez que se debilita el poder comunicativo del lenguaje, o que desaparece la comunicación lingüística, cobra especial importancia la tesis de Elaine Scarry, según la cual la inexpresabilidad del dolor personal vuelve más vulnerable a la explotación, la opresión o la tortura a manos de quien detenta el poder. Si, como bien ha señalado Lucy Bending en The Representation of Bodily Pain in Late Nineteenth-Century English Culture, la apropiación del dolor físico por parte de distintas interpretaciones

6 BOURKE, Joanna: The Story of Pain: From Prayers to Painkillers, Oxford, Oxford University Press, 2014, p. 214.

7 CHAMBERLAIN, David: "Babies don't feel pain: A century of denial in medicine", $J$ Prenat Perinat Psychol Health, 14, (1999), p. 155.

8 Esta idea ha sido desarrollada en el contexto de PICHEL, Beatriz: The emotional body: photographic, medical and theatrical practices at the turn of the $19^{\text {th }}$ century in France, Wellcome Trust, Photographic History Research Centre, Montfort University. Asimismo esta idea será desarrollada en el workshop, Emotional Bodies: A Workshop on the Historical Performativity of Emotions, Ginebra, 20-22 octubre, 2014.

9 Elaine Scarry insiste en que el poder puede ignorar el dolor de quienes no tienen la capacidad de expresarse, redundando en una mayor vulnerabilidad ante todo tipo de abusos físicos. Martin Pernick en $A$ Calculus of Suffering presenta una crónica sobre la selección de la tipología humana que necesitaba o incluso merecía anestesia cuando ésta hizo su entrada en la práctica médica habitual en los Estados Unidos de finales de 1840 y principios de 1850 . El estudio que Mary Poovey realiza sobre los acalorados debates acerca de la aplicación del cloroformo a mujeres parturientas no solo revela el conflicto entre la autoridad de médicos y clérigos, sino también el lugar de las embarazadas y el dilema sobre si se debe soportar el dolor pese a la disponibilidad de métodos anestésicos. Joanna Bourke ha señalado en dos recientes publicaciones sobre la historia del dolor, "Prothero Lecture:What is Pain?" y The Story of Pain, la importancia del estudio de las políticas del dolor. 
abre la puerta a potenciales abusos por parte de las autoridades, el dolor no solo está sometido a condicionantes culturales; también es susceptible de ser representado o deformado por dichos grupos de poder. La alianza de mecanicismo y logocentrismo en la ciencia moderna explica una visión del no hablante como no sujeto y objeto de estudio científico determinado causalmente, como se verá elocuentemente con el éxito histórico del conductismo. Aunque se han dedicado muchos esfuerzos al estudio del dolor animal, aún se ha escrito muy poco sobre las formas históricas del sufrimiento infantil.

Pese a que este estudio se centra en la cultura anglosajona, los movimientos que describe se hicieron notar a lo largo de Europa y América. Hubo notables diferencias regionales en la interpretación y la prioridad de los problemas tematizados, pero se aprecia una notable unanimidad en la importancia del estudio de la infancia.

\section{La negación del dolor}

En 1877, la revista británica de psicología y filosofía Mind publicó un breve artículo titulado "A Biographical Sketch of an Infant". El texto, escrito por un padre sobre su hijo "Willy", empezaba de la siguiente manera:

Durante los primeros siete días, mi hijo realizó sin problemas diversos actos reflejos, concretamente estornudar, hipar, bostezar, estirarse y, por supuesto, succionar y llorar. Al séptimo día, le toqué la planta del pie con un trozo de papel y él lo apartó, doblando al mismo tiempo los dedos, como haría un niño mucho mayor al hacerle cosquillas. La perfección de estos movimientos reflejos muestra que la extrema imperfección de los movimientos voluntarios no se debe al estado de los músculos ni a los centros de coordinación, sino al asiento de la voluntad. ${ }^{10}$

Con la pretensión de evaluar las distintas conductas y reacciones del pequeño, el autor de la biografía se adentra en el reino de los instintos, diferenciando lo innato de lo adquirido, y a la vez exponiendo un planteamiento según el cual el desarrollo implica un proceso de jerarquización, con un aumento gradual de la complejidad de la conducta. Así, a lo largo del texto se van tejiendo dos polaridades: la del niño como adulto en formación, que a su vez impone la comparación entre hombre y animal, y la del adulto como hombre de voluntad. Unas líneas más abajo, el escritor describe la primera visita de Willy al zoo, a la edad de dos años. Mientras que la contemplación de los animales conocidos, como el antílope y el ciervo, producen gran entusiasmo en el niño, la visión de las "bestias en casas" genera en el pequeño gran inquietud y alarma. Al no poder explicar el temor del niño en base a experiencias anteriores, el padre se pregunta si el vago pero real temor de su hijo no se deberá a "los efectos heredados de los peligros reales y las supersticiones abyectas durante tiempos salvajes en la antigüedad". ${ }^{11}$

\footnotetext{
10 DARWIN, Charles: “A Biographical Sketch of an Infant”, Mind, 2, (1877), p. 285.

11 Ibid.
} 
La importancia de esta pregunta y la repercusión de la publicación en la que se enmarca, radica tanto en su contenido como en la identidad del autor, el naturalista Charles Darwin, que, inspirado por la publicación de De l'acquisition du langage chez les enfants et dans l'espèce humaine, del filósofo francés Hippolyte Taine, decide retomar las notas de sus cuadernos "M" y "N" sobre el desarrollo de su hijo William. ${ }^{12}$ La publicación de Darwin se enmarca en la eclosión, a mediados del siglo XIX, de los primeros estudios sistemáticos del desarrollo humano en relación con las llamadas "biografías de infantes". En los 37 años transcurridos desde el nacimiento de Willy en 1840 y la publicación del texto se había producido un cambio: el funcionamiento interno de la mente infantil se convirtió, por vez primera, en objeto del interés explícito de múltiples perspectivas culturales y científicas, desde textos novelísticos y autobiográficos hasta tratados psiquiátricos y estudios de caso. ${ }^{13}$ Es cierto que desde finales del siglo XVIII, ya existían innumerables tratados pedagógicos dedicados a la educación de niños, pero el territorio de la psique infantil permaneció ajeno hasta mediados del XIX, cuando escritores y científicos comenzaron a preocuparse por explorarlo y definirlo. Hasta cierto punto, este desplazamiento se puede explicar con el cambio de la estructura social, caracterizado por la aparición de las clases medias y la escolarización creciente, procesos que abrieron nuevos espacios sociales a los niños y expandieron el periodo vital conocido como infancia. Pero existe otra razón importante. A finales del dieciocho también irrumpieron en escena las ciencias históricas, que supusieron el desembarco de modelos epistemológicos concomitantes en virtud de los cuales la realidad natural y social se comprendía como resultado de procesos de desarrollo histórico. Esta irrupción epistémica fue responsable de actitudes diferentes hacia la infancia, considerada en consecuencia el factor clave para comprender la forma adulta y el momento fundacional para la edificación del futuro de la persona. ${ }^{14}$

En la narración de Darwin, al igual que en otras biografías de la misma temática, el niño aparece como un objeto de conocimiento a través del cual se relata la historia del desarrollo humano o de la humanidad por venir. Tal como ha señalado Claudia Castañeda, el tema de fondo que se rescataba en la biografía del niño recién nacido no solo era el yo individual, sino la naturaleza humana en cuanto tal. ${ }^{15}$ Las observaciones sobre el desarrollo físico y mental de "Willy", articuladas en torno a la expresión, y que abarcan el enfado, el miedo, el afecto y el sentido moral, pusieron en marcha la investigación que culminaría en la publicación de La expresión de las emociones en el hombre y en los animales. Siguiendo aportaciones teóricas de John Locke y Jean-Jacques Rousseau sobre la infancia, Darwin la considera la única etapa natural y no cultural de la vida, página en blanco que escondía dos potencialidades. Primero,

12 LORCH, Marjorie y HELLAL, Paula: "Darwin's "Natural Science of Babies"', Journal of the history of the neurosciences, 19, 2, (2010), p. 140.

13 Véase SHUTTLEWORTH, Sally: The Mind of the Child: Child Development in Literature, Science, and Medicine, 1840-1900, Oxford, Oxford University Press, 2010.

14 Véase ZELIZER, Viviana: Pricing the Priceless Child: The Changing Social Value of Children, New York, Basic Books, I985.

15 CASTAÑEDA, Claudia: Figurations: Child, Bodies, Worlds, Durham, Duke University Press, 2002, p. 13. 
la posibilidad de moldear al futuro adulto; y segundo, más interesante para Darwin, la posibilidad de encontrar los ancestros del ser humano en el comportamiento y las expresiones del niño. Al no conceder al niño los atributos humanos de la libertad y el libre albedrío, Darwin lo clasificaba junto a los animales, sujetos perfectos en los que llevar a cabo una investigación que distinguiera entre expresiones universales (o naturales) y expresiones aprendidas. A pesar de que el cuerpo del niño estaba constituido por los mismos músculos que permitían a los adultos expresar sus emociones, la ausencia tanto de control consciente como del condicionamiento propio de la experiencia permitían entrever en el niño las expresiones instintivas del ser humano y los rasgos compartidos con los animales. ${ }^{16}$ De todas las emociones de su hijo William, Darwin dio al llanto una importancia central para sus investigaciones por ser una de las primeras expresiones en manifestarse, y también una de las más comunes. Para este prolífico observador los "gritos violentos y prolongados" de los niños eran expresiones emocionales asociadas con "el dolor, el hambre moderada o la desazón". ${ }^{17}$ Pero estos signos no provenían de la razón o el entendimiento, sino que, al igual que en el caso de los animales, los salvajes y los locos, sus lloros y gritos eran simplemente actos reflejos reforzados por la costumbre, y por lo tanto marcadores del dolor menos fiables. ${ }^{18} \mathrm{~A}$ pesar de que ya se habían publicado estudios experimentales sobre el comportamiento infantil casi dos décadas antes del registro de Darwin, este tuvo un efecto estimulante en el estudio científico de los niños, y en última instancia propició la cristalización de las teorías sobre la negación del dolor infantil. Siguiendo la publicación de El origen de las especies de Darwin (1859), y más concretamente de la mano de la emergencia de la psicología y la psiquiatría evolutivas, se produjeron pronunciados desplazamientos en la construcción de la infancia. La duradera noción popular de que el niño es como un animal o como un salvaje recibió una aparente validación científica en las teorías de la recapitulación, que veían en el niño un espejo de las formas ancestrales de las especies, tanto humanas como animales. Si Darwin sostenía en el Origen de las Especies que el dolor y la lucha eran elementos que rigen el proceso evolutivo, en esta ocasión resaltaba la centralidad del dolor en la constitución del sujeto civilizado.

Al igual que para Darwin, para el internista alemán Adolf Kussmaul, las discusiones sobre el desarrollo normal de la infancia eran un medio para comprender cómo se constituía la mente adulta. Al tiempo que Darwin tomaba las notas que conformarían el texto "Esbozo biográfico de un infante", Kussmaul publicó un informe sobre el comportamiento y el repertorio sensorial de los recién nacidos. ${ }^{19}$ Las discusiones sobre el desarrollo normal durante la primera infancia eran parte esencial de sus clases de psiquiatría en la Universidad de Heidelberg, y fue precisamente su insatisfacción hacia la bibliografía existente lo que empujó a Kussmaul a llevar a cabo sus propios

16 PRODGER, Philip: Darwin's Camera, Oxford, Oxford, University Press, 2009, p. 105.

17 DARWIN, Charles: The Expression of Emotions in Man and Animals, London, John Murray, 1872, p. 147.

18 RODKEY, Elissa y PILLAI RIDDELL, Rebecca, p. 343.

19 KUSSMAUL, Adolf: Untersuchungen uber das Seelenleben des Neugeborenen Menschen. Leipzig, DE, Winter, 1859. 
experimentos. ${ }^{20} \mathrm{Su}$ investigación se centró en la función de los sentidos, incluyendo el tacto, la sensibilidad a la temperatura, experimentos sobre el olfato, experimentos visuales, experimentos auditivos y "experimentos sobre el dolor, la sensibilidad muscular y el hambre de aire". ${ }^{21}$ A diferencia de las demás secciones, la de la sensibilidad al dolor no incluía resultados experimentales. La reticencia de Kussmaul a experimentar con el dolor se debía a que los experimentos parecían causar molestias en el niño. Los llantos y la inquietud que mostraban los niños después de los ensayos experimentales con ácido tartárico le llevaron a interrumpir este experimento en concreto. Tras Kussmaul, el desarrollo de los estudios sobre la infancia se acelera, con un gran volumen de contribuciones en pocos años. En 1873, Alfred Genzmer defiende su tesis doctoral, con ocasión de la cual realiza un estudio crítico de la obra de Kussmaul. ${ }^{22}$ Pero si la discusión de Kussmaul, al igual que la de Darwin, se basaba en observaciones más incidentales que experimentales, Genzmer produjo deliberadamente dolor en los niños, pinchándoles con alfileres en la nariz, el labio superior y las manos. A pesar de que la intensidad del estímulo era tal que "salían pequeñas gotas de sangre de los pinchazos", afirmaba que los niños "no mostraron ningún indicio de malestar, ni siquiera un leve estremecimiento ${ }^{23}$, aun reconociendo que el estímulo iba precedido de expresión, pues "la humectación de los ojos" aumentaba al clavar las agujas en las caras de los niños. Con todo, concluía que el dolor está "excepcionalmente poco desarrollado en el neonato". ${ }^{24}$ En el trabajo de Genzmer el dolor se presenta como una herramienta del método experimental, y los niños son objetos de experimentación ligados a las investigaciones sobre el desarrollo de las funciones orgánicas.

Unos años más tarde, el trabajo de Genzmer fue continuado por Traugott Kroner, quien pronunciaba en 1882 una lección inaugural en la universidad de Breslau sobre el mismo tema, que se fue afianzado progresivamente. ${ }^{25} \mathrm{El}$ científico Silvio Canestrini siguió esta línea de investigación y empleó diferentes estímulos sobre los cinco sentidos a un grupo de setenta niños durante fases de sueño y vigilia. En este caso, se examinaron las respuestas cerebrales del niño a distintos estímulos mediante el ajuste de un aparato creado al efecto: una variante del pneumógrafo de Marey, que se aplicaba a la fontanela del niño para medir, al mismo tiempo, el pulso cerebral y la respiración. Los resultados de los experimentos de Canestrini corroboran parcialmente los hallazgos de Kussmaul y Genzmeer, pero también llegan a conclusiones diferentes. Canestrini, para quien el neonato humano está claramente por debajo de las crías recién nacidas de gatos y perros, concluyó que el tacto infantil muestra una

20 BRINGMANN, Wolfgang; BRINGMANN, Norma y BALANCE, William: "Experimental Approaches to Developmental Psychology before William Preyer", en ECKARDT, Georg, Contributions to a History of Developmental Psychology: Internacional William T. Preyer Symposium, Berlin y New York, Mouton, 1985, p. 159.

21 BRINGMANN, Wolfgang; BRINGMANN, Norma y BALANCE, William: "Experimental Approaches...", p. 159.

22 GENZMER, Alfred: Untersuchugen Ueber die Sinneswahrhehumugen des Neugeborenen Menschen, Inaugural Dissertation, Halle, Plötzche Buchhandlung, 1873.

23 Ibid., p. 12.

24 Ibid., p. 22.

25 MAATER, Florence: Child behavior; a critical and experimental study of young children by the method of conditioned reflexes, Boston, The Gorham Press, 1918, p. 20. 
reacción sorprendentemente leve a los estímulos dolorosos y una escasa excitabilidad eléctrica del sistema nervioso. ${ }^{26}$ Las prácticas experimentales de Kussmaul, Genzmer, Kroner y Canestrini se enmarcan en el desarrollo de la nueva fisiología, que se desarrolló en Alemania alrededor de la figura de Johannes Müller Este nuevo cuerpo de conocimiento, que proponía un programa empírico para el estudio de las funciones vitales, tenía una fuerte dependencia del desarrollo de nuevas líneas teóricas de investigación del dolor. ${ }^{27}$ Müller y otros profesionales, como Richard Bright, Maximiliary Von Frey y A. Goldschneider, se concentraron en los nervios para describir la fisiología del dolor, lo que condujo a una comprensión cada vez más sofisticada del sistema nervioso no exenta de importantes disputas. ${ }^{28} \mathrm{El}$ análisis de las secciones del cerebro por parte de Paul Emil Flechsig, profesor extraordinario de psiquiatría en la Universidad de Leipzig, a partir de 1872, llevó al descubrimiento de la mielinización secuencial de las fibras incluso después del parto. ${ }^{29}$ Como el recién nacido presentaba tanto fibras mielinizadas como no mielinizadas, considerándose totalmente funcionales únicamente las primeras, la conclusión fue que la escasa mielinización de los recién nacidos obstaculizaba la funcionalidad plena del dolor y demás estímulos sensoriales. A la vez que se iba desarrollando esta línea de investigación, comenzó la disciplina científica que estudiaba el desarrollo infantil en su normalidad. La publicación de la obra de Darwin A biography Sketch of an Infant proclamó la emergencia en Europa de un nuevo campo de observación y experimentación que tenía por objeto al infante en sus primeros meses de vida. La aparición de la psicología infantil se puede observar en las personalidades de Wilhelm Preyer en Alemania, Bernard Perez en Francia, James Sully en Reino Unido y G. Stanley Hall en Estados Unidos.

Unos años más tarde, en 1889, el fisiólogo inglés William Thierry Preyer publicaría en Alemania Die Seele des Kindes, que resumía la observación del desarrollo de su hijo Axel durante los primeros tres años de su vida. A pesar de que la obra de Preyer se enmarcaba en la tradición de las biografías de infantes, sus páginas contienen referencias a los primeros trabajos empíricos en fisiología y psicología infantil, entre ellos los de Genzmer y Darwin. Fuertemente influenciado por la teoría de la evolución, Preyer entendía el desarrollo como una trayectoria en la que el lenguaje y la acción propositiva sustituían gradualmente el instinto y el reflejo. A pesar de que Preyer confiaba ampliamente en Genzmer como autoridad en el estudio de los recién nacidos, en la sección dedicada a "Unpleasant Feelings in General", el fisiólogo escribió: "Es un error sostener que el niño de más corta edad todavía no sea capaz de albergar la sensación genuina de dolor, o incluso un alto grado de sensación de desagrado", argumentando que "quien puede disfrutar también debe ser capaz de

26 Ibid., p 20.

27 MOSCOSO, Javier, Historia Cultural del Dolor, Madrid, Taurus, 2011, p. 122.

28 Pueden encontrarse resúmenes en REY, Roselynne: The History of Pain, Cambridge, MA, 1995; MELZACK, Ronald y WALL, Patrick: "Pain Mechanisms: A New Theory", Science, 150, 3699, 1965, p. 971-9, y LESCH, John, Science and Medicine in France. The Emergence of Experimental Physiology, 17901855, Cambridge, Mass. y London, Harvard University Press, 1984.

29 La conferencia de Paul Flechsig, resumida por Frederick W. Mott, puede encontrarse en 'Cerebral Development and Function', British Medical Journal, 1.3145, 9 Abril 1921, 529. En BOURKE, Joanna: The Story of Pain: From Prayers to Painkillers, Oxford, Oxford University Press, 2014, p. 216. 
sufrir, pues de lo contrario no podría disfrutar." ${ }^{30}$ Si bien Preyer admitía que el recién nacido es menos sensible a las impresiones dolorosas que los adultos, según él "sería erróneo inferir de ello una condición de anestesia o analgesia." ${ }^{31} \mathrm{Al}$ igual que su hijo Axel, que "reaccionaba con movimientos frente al roce más leve de su cara", Preyer pensaba que los niños mostraban indicios de experimentar dolor, y declaraba que era algo "inconfundible para cualquier observador diligente. Sobre todo, el llanto es característico: es desgarrador y persistente en el dolor". ${ }^{33}$ La conclusión de Preyer es matizada; por un lado, niega que los niños experimenten el dolor como los individuos adultos, por el otro evita concluir que no experimenten ningún dolor, porque "es posible provocar gritos y movimientos en niños y animales recién nacidos... pellizcándoles la piel". ${ }^{34}$ La interpretación de Preyer del placer y del dolor como instintos básicos del niño, así como la comparación del infante con el animal, era común a finales del siglo XIX, y aún a principios del XX, y tenía su origen en el darwinismo como parte de un proyecto de mayor envergadura para explicar el comportamiento en términos adaptativos. Experimentar placer y evitar el dolor se erigieron como las dos motivaciones primarias para la acción, lo que llevó a que autores de diarios, como la psicóloga estadounidense Milicent Washburn Shinn, cuyo libro The Biography of a Baby se publicó en 1900, pudieran interpretar inconscientemente sus observaciones desde este eje. Al contrario que Preyer, cuyo hijo tenía una sensibilidad "sorprendentemente alta" en la piel, Shinn mantenía del suyo que "en sus primeros días no tuvo experiencia alguna de dolor en la piel, y al ser mantenido a una temperatura templada, probablemente tampoco recibiera sensaciones definidas de frío ni calor" ${ }^{35}$. El dolor se presenta en el relato como una sensación que el niño va aprendiendo a través de la experiencia y que va ligada al proceso evolutivo y de conciencia de sí. El antropólogo inglés Alexander Francis Chamberlain también defendía que los niños poseen el instinto de la conservación, "como si sintieran la fragilidad de la existencia y se agarraran a ella con todas sus fuerzas; esto es cierto fisiológica y psicológicamente." ${ }^{36}$ Según Chamberlain, la falta de sensibilidad ante dolores, golpes y heridas durante los primeros años de edad es, a menudo, sorprendente:

$\mathrm{Al}$ no ser capaces de localizar el dolor con prontitud suficiente hasta el segundo o tercer año de vida, en su resistencia al dolor físico y en su desesperado aferrarse a la vida se parecen a los salvajes. ${ }^{37}$

El texto de Chamberlain participa, además, de la creencia según la cual las culturas menos evolucionadas tenían mayor resistencia al dolor. Unos años más tarde,

30 PREYER, William: The Mind of the Child: The development of the intellect, New York, Arno Press, 1889, p. 147.

31 Ibid., p. 147.

32 Ibid., p. 105

33 Ibid., p. 147

34 Ibid., p. 97

35 SHINN, Milicent: The biography of a baby, Boston y New York, Houghton Mifflin, 1900, p. 47.

36 CHAMBERLAIN, Alexander Francis: The child: a study in the evolution of man, London, W. Scott Ltd., 1900 , p. 337

37 Ibid. 
el psicólogo americano Stanley Hall publicaría el primer estudio sistemático de la mente infantil realizado en Norteamérica, Aspects of child life and education. Profundamente interesado en la psicología evolutiva y en el desarrollo infantil, Hall estaba fuertemente influido por la teoría de la recapitulación de Ernst Haeckel, quien sugirió que las etapas embrionarias de un organismo se asemejan a las fases en el desarrollo evolutivo. Por lo tanto, al igual que la obra de Shinn, su estudio del niño y el adolescente siempre se condujo en el marco estructural de la evolución biológica y cultural. Hall, que también entendía el dolor y el placer como las afectividades fundamentales y primarias del niño, defendía que si bien la autoconciencia física comienza con el reconocimiento de la mano y, más tarde, del pie, "generalmente la autoconciencia psíquica lo es solo del dolor, ya sea interno, como el dolor de estómago, o periférico, como los cortes y golpes". ${ }^{38}$ La figura de Stanley Hall ejerció un efecto profundo sobre la psicología estadounidense, en particular en las ramas de la psicología infantil y el desarrollo adolescente. Muchos de los estudiantes de Hall llegaron a liderar la nueva disciplina de la psicología infantil. Entre ellos se encontraba, en especial, Arnold Gesell, quien no solo adoptó plenamente la visión de su mentor sobre la teoría de la recapitulación, sino que llegó a acuñar el nuevo término de "maduración", en el cual se reflejaba la integración de crecimiento fisiológico y social que había defendido Hall. La obra de Gesell ayudó a que cristalizase en el siglo XX la idea del dolor infantil como un acto reflejo, "raudo pero inmaduro", si bien afirmaba que "la sensibilidad a los estímulos dolorosos aumenta de forma definitiva en la primera semana tras el parto". ${ }^{39}$

A medida que la psicología evolutiva iba dejando de ser el paradigma dominante, el conductismo aplicó a la práctica clínica las nuevas teorías científicas basadas en las ideas de Darwin y en los datos anatómicos e histológicos de la embriología y la fisiología, reforzando la idea de que infantes y niños eran relativamente insensibles al dolor. Los experimentos sobre sensibilidad y dolor infantil hicieron su aparición en la historia de la psicología conductista de la mano de Margaret Gray Blanton, una especialista en el campo de la logopedia. Blanton realizó una serie de experimentos en la Universidad Johns Hopkins de Baltimore bajo la supervisión del psicólogo John Broadus Watson, considerado padre fundador del conductismo. Watson, que estaba especialmente interesado en el estudio del modelo de estímulo-respuesta del condicionamiento clásico y su aplicabilidad a la predicción y el control de la conducta humana, se centró en lo que según él eran las tres emociones humanas fundamentales: el amor, la ira y el miedo; su discípula, sin embargo, se centró en el estudio del dolor. La investigación del dolor encajaba particularmente bien en los valores de la psicología conductista, ya que las expresiones ante el dolor podían interpretarse como una mera respuesta mecánica al estímulo. Blanton analizó las reacciones de un grupo de neonatos ante estímulos lesivos, tales como la extracción de sangre y pinchazos en las muñecas durante el sueño. Los infantes reaccionaron con actitudes defensivas a los experimentos: las sajaduras provocaban llantos exacerbados, los pinchazos durante el

38 HALL, Stanley: Aspects of child life and education, New York y London, D. Appleton and company, 1921, p. 30.

39 GESELL, Arnold: Infant behavior: Its genesis and growth, New York and London, McGraw-Hill Book Company, 1934, p. 281. 
sueño hacían que la mitad de los infantes movieran la mano y el antebrazo, la frotación brusca de la espalda y la cabeza para eliminar la vérnix provocaba movimientos de las manos de vigorosa resistencia, un afán frenético por escapar y llantos de enfado. Sin embargo, Blanton se resistió a extraer la conclusión de que es el procedimiento médico lo que provocaba el llanto, defendiendo que, al ser expresiones propias de la conducta infantil, no se podía inferir ninguna conclusión de dichas reacciones. ${ }^{40}$ Finalmente, concluyó que "el reflejo y el equipamiento instintivo del infante al nacer es más complejo y avanzado de lo que se creía hasta el momento". ${ }^{41}$

La progresiva sofisticación de los experimentos conductistas condujo a un control más exacto de la situación experimental. Esto resultó en la sustitución de los estímulos lesivos previos por la introducción de descargas eléctricas en los experimentos, ya que la descarga eléctrica eliminaba la variabilidad de la presión de la aguja. Esta línea de investigación siguió con una serie de experimentos en la Universidad Northwestern y en el Hospital Lying-In de Chicago a cargo de Mandel e Irene Sherman. ${ }^{42}$ Si bien virtualmente todos los infantes reaccionaron durante las primeras horas y el primer día después del nacimiento a dichos estímulos, los investigadores concluyeron que la tendencia era de más reacción frente a menos estimulación desde el primer día al duodécimo. Como descubrimiento fisiológico, esto sugería que, al nacer, los recién nacidos no eran muy sensibles, pero que su sensibilidad aumentaba gradualmente. ${ }^{43} \mathrm{Al}$ igual que hiciera Blanton, los Sherman registraron que los niños intentaban escapar y realizaban movimientos defensivos con los brazos y piernas ante estos experimentos, incluyendo golpes al objeto para alejarlo, pero dudaban de qué conclusiones extraer sobre el dolor infantil a partir de su propio experimento: "Tal vez las respuestas de los infantes a los estímulos dolorosos no puedan llamarse respuestas al dolor, ya que se desconoce su naturaleza psicológica". ${ }^{44}$ El hecho de que los experimentos de los Sherman se publicaran en The Journal of Comparative Psychology y en Comparative Psychology Monographs en el contexto de experimentos parecidos que usaban descargas en animales y registraban los "reflejos" resultantes, sugiere la visión mecanicista dominante de los niños de menor edad..$^{45}$ Junto con los animales, los infantes constituían los objetos pasivos perfectos para los experimentos que se decantaban por una perspectiva conductual reduccionista, ya que los investigadores buscaban reflejos y no conciencia. En este contexto, la interpretación de los Sherman no fue discutida por nadie, lo que allanó el camino para experimentos rutinarios sobre los umbrales del dolor infantil sin necesidad de ninguna justificación ética especial. La presentación de los Sherman fue un indicador de lo que llegaría a continuación: a medida que el conductismo perdía su monopolio en el pensamiento psicológico, la

40 BLANTON, Margaret Gray: "The behavior of the human infant in the first 30 days of life", Psychol Rev, 24, (1917), p. 472.

41 Ibid.

42 SHERMAN, Mandel y SHERMAN, Irene: "Sensori-motor Responses in Infants", Journal of Comparative Psychology, 5 (1925), p. 53-68; SHERMAN, Mandel y SHERMAN, Irene: "Infant behavior", Comparative Psychology Monographs, 12, 1-107, (1936).

43 SHERMAN, Mandel y SHERMAN, Irene: "Infant behavior", p. 8.

44 Ibid., p. 38

45 RODKEY, Elissa y PILLAI RIDDELL, Rebecca, p. 345. 
maduración cerebral se convirtió en el foco dominante de la investigación sobre el dolor de los niños.

\section{El lenguaje del dolor}

En 1833, en una reseña de un pionero texto francés sobre enfermedad infantil, un contribuidor a The Lancet reconocía que, pese al progreso general de la medicina, generalmente las enfermedades infantiles habían sido ignoradas o atribuidas a la dentición, a la presencia de gusanos o a una vaga noción de "crecimiento". ${ }^{46} \mathrm{Si}$ bien médicos anteriores ya habían reconocido una serie de enfermedades que, o bien eran muy comunes durante la infancia, o bien eran consideradas exclusivamente infantiles, el estudio de las patologías propias de este periodo de la vida solo se desarrolló como especialidad médica en la segunda mitad del siglo XIX. La búsqueda de reconocimiento profesional de los anestesistas a finales del siglo XIX, y del interés por parte de la fisiología y la psicología evolutiva por el desarrollo del niño, coincidió con las aspiraciones de legitimidad de otro grupo profesional: los médicos infantiles. Así, esta especialidad médica, basada en la construcción científica de la dicotomía niño/ adulto, comenzó a organizar el cuidado de la infancia en formas más científicas. ${ }^{47} \mathrm{La}$ explicación habitual que ha recibido la génesis de la pediatría da cuenta de los cambios en la concepción, el significado y la importancia de la infancia inaugurados con el pensamiento europeo durante la Ilustración. ${ }^{48} \mathrm{Si}$ la infancia había sido considerada como un estado incompleto del ser carente de una relación plena con la vida, por lo que se consideraba un despilfarro dedicarle demasiada atención y cuidados, la aparición de teorías más optimistas sobre el desarrollo humano llevó a una percepción de la infancia como fuente del progreso futuro que debía ser gestionada con cuidado.

Fue así como, a lo largo del siglo XIX, se fueron creando en distintas ciudades europeas los primeros hospitales dedicados exclusivamente al cuidado de niños enfermos, con el importante precedente de París en $1802^{49}$. La llegada a Reino Unido se dilató, sin embargo: fue en febrero de 1852 cuando tuvo lugar la inauguración del Great Ormond Street Hospital de Londres (de ahora en adelante, GOSH). El hospital ha sido descrito muchas veces como la realización de la visión del médico Charles West, que se había formado como "Physician Accoucheur", figura a medio camino entre el ginecólogo moderno y una comadrona masculina. West se inspiró en las

\footnotetext{
46 Lancet, 1833-34, 1, p. 367, en STANLEY, Peter: For Fear of Pain: British Surgery, 1790-1850, Amsterdam-New York, Editions Rodopi, 2003.

47 Sobre la historia de la pediatría ver: A. R. Colón y P. A. Colón, Nurturing Children: A History of Pediatrics, Westport, Conn, Greenwood Press, 1999; GARRISON, Fielding y ABT, Arthur: Abt-Garrison History of Paediatrics, with New Chapters on the History of Paediatrics in Recent Times1 Philadelphia and London, W.B. Saunders, 1965.

48 LOMAX, Elizabeth: "Small and Special: The Development of Hospitals for Children in Victorian Britain”, Medical History, 16, London, Wellcome Institute for the History of Medicine, (1996), p. 1.

49 GARRISON, Fielding y ABT, Arthur: History of Pediatrics, p. 289.
} 
instituciones infantiles francesas y alemanas y en los hospitales de maternidad de Dublín ${ }^{50}$.

Cinco años antes de que el GOSH abriera sus puertas al público, West impartió a los estudiantes del Hospital de Middlesex una serie de conferencias sobre las enfermedades de infantes y niños ${ }^{51}$ que ya contienen el germen de lo que sería su futura práctica pediátrica. El texto, como muchos otros de la época, abría con el debate sobre si los niños eran capaces de comunicar sus emociones y su dolor de manera fehaciente. Las dificultades diagnósticas de muchos médicos ante pacientes infantiles generaron la creencia de que los niños eran incapaces de comunicar sus experiencias de forma fiable. Ya en 1849, Hess describía la pediatría como la más ardua de las especialidades médicas: “...debido a que los niños o son incapaces o se muestran reacios a describir adecuadamente sus sentimientos, ya sea por su inseguridad, timidez o resistencia a los procedimientos del médico". ${ }^{52}$ West sostenía apasionadamente que, pese a las importantes barreras que los jóvenes médicos se encontraban a la hora de examinar a los niños, éstos demostraban el dolor tanto comportamental como fisiológicamente, aportando pruebas suficientes para ser debidamente reconocidas por cualquier médico decente cuya praxis no se basara en el folklore o en formas vetustas de pronóstico. "No puedes preguntar nada a tu paciente", explicaba West. La falta de respuesta se podía deber, según West, a una edad demasiado temprana para hablar, pero también a la parálisis provocada por el miedo. Los médicos debían obtener información de la expresión del niño y de su comportamiento, lo que tampoco era una cuestión sencilla.

El niño es temeroso y no está dispuesto a ser examinado, intentas tomarle el pulso y se resiste, presa de la alarma; intentas auscultarle el pecho e irrumpe en llantos o en un ataque violento. ${ }^{53}$

Algunos hombres jóvenes, no familiarizados con el trato con niños y acostumbrados a constituciones más robustas, se mostraban incapaces de aprender a examinar y diagnosticar a sus pacientes más jóvenes. West los emplazaba a perseverar: aunque el infante no pueda hablar "tiene, sin embargo, un idioma propio, y ese idioma debe ser su objeto de estudio". ${ }^{54}$ Con estas palabras West no solo ponía de manifiesto las dificultades a las que se enfrentaban quienes estuvieran interesados en el tratamiento de la enfermedad infantil; también recalcaba la importancia y necesidad de la figura del pediatra. West comparaba al médico de niños con un explorador que, al entrar en un territorio cuyos habitantes suponía hablantes de la misma lengua y practicantes de los mismos usos que la gente de su propio país, se viera envuelto en los sonidos de

\footnotetext{
50 TANNER, Andrea : "Too many mothers? Female roles in Metropolitan Victorian children's hospital", en HENDERSON, John y PASTORE, Alessandro (eds.), The Impact of Hospitals, Oxford and Bern, Peter Lang, 2007.

51 WEST, Charles, Lectures on the Diseases of Infancy and Childhood, London, Longman, Brown, Green \& Longmans, 1852 .

52 HESS, A: "On the necessity of practical instruction in the treatment of diseases of children", Lancet, 10 (1849), p. 341.

53 WEST, Charles, p. $1-3$.

54 Ibid.
} 
una lengua extranjera. ${ }^{55}$ Mientras que algunos profesionales nunca superaban estas dificultades, motivo por el cual las enfermedades infantiles se volvían para ellos un libro cerrado, el buen médico se dedica con paciencia a estudiar "una nueva semiología, una nueva patología y una nueva terapéutica", accediendo gracias a este nuevo lenguaje a la confianza del niño y a su enfermedad. ${ }^{56}$ West, que usaba la metáfora del explorador para dar sentido a la falta de una historia del paciente en primera persona, defendía que quienes no cultivasen sus facultades de observación nunca podrían aprender el lenguaje de la infancia. West iba un paso más allá, comentando a sus estudiantes cómo aquellos médicos que no apreciasen a los niños pequeños no podrían aprender este lenguaje, "ya que pronto perciben quién los ama, y si caen enfermos no expresarán a nadie más sus sentimientos reales, ya sea con palabras o con signos." ${ }^{7}$ Quienes estuvieran interesados en el estudio práctico y teórico de las enfermedades infantiles no solamente tenían que estar dispuestos a escuchar y entender a sus pequeños pacientes; también debían quererlos. Thomas Rotch, primer catedrático de Pediatría en Harvard, parafraseó a West en 1891 con ocasión de su discurso presidencial en la American Pediatric Society al describir la experiencia de un infante ante sus colegas: "Nos hemos adentrado en la investigación especial en esta rama de la antropología con los intereses sagaces de exploradores en un país casi desconocido". ${ }^{58}$

Como ponen de manifiesto las narrativas de West y de Rotch, diagnosticar correctamente la enfermedad infantil fue un desafío al que se enfrentaron muchos médicos, motivo por el cual el dolor fue un asunto tratado con gran seriedad por los médicos interesados en el campo de la salud infantil. Reducido por la comunidad científica a poco más que síntoma de una enfermedad o una lesión, a finales del siglo XIX el dolor se reivindica por su valor diagnóstico. El médico irlandés Richard Tonson Evanson ponía de manifiesto a mediados del siglo el uso instrumental del dolor en la sección "Diagnosis" de su manual sobre enfermedades infantiles. Para Evanson era muy necesario determinar la existencia del dolor y su localización en el niño, o la causa que pueda provocar el llanto infantil, ya que el dolor y los gestos expresivos que lo acompañan eran la manera más directa de acceder a la enfermedad. ${ }^{59} \mathrm{Como}$ ya señalara West, la importancia de los gestos físicos del niño y la traducción de dichos gestos en signos clínicos podían conferirles carta de lengua, permitiendo a estos médicos acercarse al dolor en la infancia. Médicos como West y Evanson alentaban los gritos y los llantos de los niños no porque no pudieran remediarlos, sino porque creían que, al promover la abundancia de estos signos, podían alcanzar un diagnóstico más preciso de la enfermedad. Esta semiótica hizo que, hacia finales del siglo XIX, al mismo tiempo que Darwin intentaba conectar las expresiones de los niños con sus emociones y los fisiólogos el dolor con el sistema nervioso, los médicos inte-

55 Sobre las metáforas de la pediatría véase: GILLIS, Jonathan, y Loughlan, Patricia: "Not just small adults: the metaphors of paediatrics", Arch Dis Child, 92, 11 (2007), pp. 946-947.

56 Ibid.

57 Ibid.

58 ROTCH, Thomas: "Iconoclasm and original thought in the study of pediatrics", Arch Pediatr, 7, (1891), p. 811.

59 EVANSON, Richard Tonson: A practical treatise on the management and diseases of children, Dublin, Fannin \& Co, London, Renshaw and Longman, 1842, p. 63. 
resados en la infancia intentaran conectar los signos del dolor infantil con las lesiones orgánicas. De esta manera comenzaron una búsqueda más sistemática de las pruebas del dolor en niños, ampliando las observaciones sobre el llanto infantil y examinando los cambios en la expresión facial de los niños o los hábitos de sueño bajo los efectos de la enfermedad.

Al concentrar el análisis del dolor en los síntomas corporales y en la modificación de la conducta, muchos médicos sortearon la problemática acerca de la capacidad infantil para comunicar sus propias experiencias de forma fiable. Pero otros médicos se interesaron por el valor comunicativo del llanto: "El llanto es el medio más importante, si no el único, de que dispone el pequeño infante para indicar disgusto, desazón o sufrimiento", declaraba el médico estadounidense Louis Starr, que consideraba que los niños sanos lloran en raras ocasiones. ${ }^{60} \mathrm{~A}$ pesar de que el llanto parece ser el comportamiento más veces asociado con el dolor, existían controversias acerca de si el llanto siempre indicaba desazón, dolor o enfermedad en un niño. Por este motivo, los médicos se esforzaron en desarrollar un esquema de evaluación del dolor infantil basado en distintos tipos de llanto. Evanson pensaba que el llanto que procedía de un sufrimiento agudo "es claro, alto, sonoro y continuo", perteneciendo más a la expiración que la inspiración. ${ }^{61}$ El médico irlandés añadía al llanto una serie de gestos complementarios, como la disposición de las cejas y de las manos, intentando construir una conexión invariable entre el signo y la lesión anatómica. Para Starr, primer catedrático de Pediatría de la Escuela de Medicina de la Universidad de Pennsylvania, era posible establecer una relación directa entre distintos tipos de llanto y determinadas enfermedades y gradaciones del dolor. Mientras que el llanto hidrocefálico, consistente en un grito súbito, paroxístico y de gran volumen, implicaba dolor de cabeza, Starr entendía que un llanto alto y sin interrupciones precedía un ataque espasmódico, mientras que si el niño lloraba durante un ataque de tos o poco tiempo después y el llanto iba acompañado de la deformación de las facciones, sufría neumonía ${ }^{62}$ El pediatra estadounidense Luther Holt, especialmente interesado en que el dolor de los niños menores de dos meses no fuera ignorado, defendía que la falta de lágrimas antes de este tiempo no debía tomarse como una indicación de que el pequeño no sintiera dolor. Holt dividió los llantos infantiles en distintos grupos funcionales según la causa fuera el hambre, la indigestión, el humor, la debilidad, el cansancio, la incomodidad, el dolor o el hábito. Holt sugirió que los niños que sufrían un dolor extremo lloraban de forma aguda y penetrante, contraían los rasgos faciales, elevaban las piernas y, en ocasiones, terminaban por caer presa de un sueño exhausto. ${ }^{63}$ En su opinión, el llanto a causa de dolores menos severos era un gimoteo de menor volumen. Ralph Vincent, fundador de The Infants' hospital en Westminster, también

60 STARR, Louis, "The clinical investigation of disease and the general management of children", en STARR, Louis y WESTCOOTE, T. S, (eds), An American text-book of the diseases of children, Philadelphia, Saunders, 1985, p. 3.

61 EVANSON, Richard Tonson: A practical treatise on the management and diseases of children, 4th ed, Dublin: Fannin \& Co, London: Renshaw, and Longman, 1842, p. 108.

62 STARR, Louis, p. 6.

63 HOLT, Luther: The diseases of infancy and childhood: for the use of students and practitioners of medicine, New York, Appleton, 1987, p. 1-45. 
afirmaba que el llanto producido por un dolor intenso era característico: "es agudo y penetrante y está unido a la contracción de las extremidades y de las facciones." Unas líneas más abajo añade: "uno de los llantos que se malinterpreta con mayor frecuencia es el que se debe al dolor de la indigestión. El llanto en sí mismo no es tan agudo como el que se debe a dolores más intensos, y se acerca mucho al llanto por hambre". ${ }^{64} \mathrm{Al}$ igual que para Darwin existía una relación directa entre las emociones y sus expresiones, para el médico Starr el dolor también se expresaba siempre a través de los mismos gestos y las mismas expresiones. Starr describió la expresión facial del dolor infantil e indicó cómo inferir el origen del dolor de estas expresiones. Por ejemplo, este médico defendía que mientras "la contracción del ceño denota dolor de cabeza", el estiramiento de las fosas nasales denotaba "dolor pectoral, y la elevación del labio superior, dolor abdominal". ${ }^{65}$

Pero no todos los autores concordaban con Starr en esto. Aun cuando los manuales sobre condiciones infantiles publicados en la segunda mitad del siglo XIX nunca cuestionaron el valor diagnóstico del dolor, autores como el norteamericano John Forsyth Meigs, uno de los pioneros en el estudio de la enfermedad infantil, criticaron la creencia de que el origen del dolor se pudiera localizar a partir de la respuesta del paciente. ${ }^{66}$ Aquellos médicos que repudiaban la concepción que asociaba determinadas patologías con sus signos expresivos, subrayaron la importancia de obtener una buena historia clínica. De esta manera James Carmichael, médico en el Royal Hospital for Sick Children, aconsejaba a sus colegas de profesión

hablar con la madre o la enfermera y descubrir la historia de las enfermedades actuales y pasadas, el número de hijos y la posición del paciente en la familia; el número y la salud de los que estén vivos; las causas de defunción de todos los que hayan muerto; la salud de la madre durante el embarazo; la naturaleza del parto, así como cualquier dificultad o anomalía detectadas tras el alumbramiento; el número de embarazos malogrados y el modo de alimentación. ${ }^{67}$

Estas investigaciones preliminares se asemejaban al método diagnóstico explicado por West en Lectures on the diseases of infancy and childhood, practicado de forma casi literal por todos los pediatras del GOSH. Pese a la desconfianza del gremio médico hacia la madre ${ }^{68}$, el relato materno era la fuente primaria para la construcción de la historia clínica de las enfermedades infantiles.

A comienzos del siglo XX los médicos seguían lidiando con la dificultad de determinar los síntomas de la enfermedad infantil, y figuras como la de David Forsyth, médico en el Evelina Hospital for Sick Children, todavía describían el dolor como uno de "los muchos síntomas de la enfermedad", haciendo hincapié en que "ningún

${ }^{64}$ VINCENT, Ralph: The nutrition of the infant, 4th ed, London, Bailliere, Tindall and Cox, 1913, p. 198.

65 STARR, Louis, "The clinical investigation of disease...", p. 3-4.

66 MEIGS, John Forsyth: A Practical Treatise on the Diseases of Children, Philadelphia, Lindsay and Blackiston, 1853, p. 23.

67 CARMICHAEL, James: Disease in children: a manual for students and practitioners, Edinburgh, Pentland, 1892, p. 3.

68 TANNER, Andrea, "Too many mothers?.., p. 55. 
otro puede rivalizar con su valor diagnóstico". ${ }^{69}$ Como muchos otros autores que han glosado los diagnósticos de enfermedades infantiles, a Forsyth le preocupaba sobremanera la dificultad que los niños tenían de comunicar un objeto tan escurridizo como el dolor. Recordaba a sus lectores que

En las edades en que los niños carecen de la capacidad de expresión verbal, no siempre es fácil reconocer la presencia, la naturaleza o la intensidad del dolor, y aún más complicado resulta determinar su localización o trazar su distribución. Esto no se debe solamente a la incapacidad para el habla del infante, sino a su propia ignorancia sobre el lugar del dolor. ${ }^{70}$

Con todo, estos problemas distaban de ser exclusivos de los niños de más corta edad, ya que los médicos tampoco confiaban en los testimonios de niños de más edad: aunque un niño sea capaz de hablar, "es bastante incapaz de describir los síntomas o localizar el dolor con una precisión fiable," afirmaba Forsyth. Otro médico especializado en enfermedades pediátricas y pionero en el estudio de infantes en instituciones, el estadounidense Henry Dwight Chapin, volvía a defender, en 1911, que las distintas partes del rostro infantil delataban la localización del dolor en ciertas partes del cuerpo. La parte superior de la cara apuntaría a dolores y enfermedades de la cabeza; la alteración del semblante en la parte media del rostro estaría relacionada con dolores pectorales; y la parte inferior del rostro sería una expresión de dolor en los órganos abdominales. ${ }^{71}$ El mismo año, el obstetra Pierre Budin, avisaba a las madres sobre cómo "detectar la enfermedad en su primera aparición, y por lo tanto garantizar al niño la oportuna asistencia médica". ${ }^{72}$ Al igual que Holt, Budin defendía que, a través de la expresión, la madre podía realizar un diagnóstico del niño. Si las cejas se presentaban contraídas, se denotaba que el niño tenía dolor en la cabeza. Este gesto se consideraba con frecuencia el primer signo exterior de que algo iba mal, y como afirmaba Budin siempre ocurría en el "mismísimo arranque de la enfermedad". ${ }^{73} \mathrm{Si}$ este signo no era identificado por la madre, y la enfermedad proseguía su curso, pronto "los ojos se detienen y comienzan a mirar fijamente." Más tarde vendría el rechinar de dientes, los movimientos de pies y manos, y por supuesto el llanto. Mientras que si los labios estaban separados de modo que el médico podía ver los dientes o las encías del niño, el dolor estaba localizado en la barriga, la dilatación de las fosas nasales implicaba dolor en el pecho. Si bien los tratados anteriores defendían que la combinación de amor hacia el niño más la capacidad de sympathein, hacían que una madre con la debida composición afectiva pudiera ser mejor que el más inteligente de los pediatras, estos tratados ya no buscan aprender del relato materno, sino enseñar a las madres cómo llevar a buen término el cuidado de sus hijos.

\footnotetext{
69 FORSYTH, David: Children in health and disease: a study of child-life, London, John Murray, 1909, p. 300 .

70 Ibid.

71 CHAPIN, Henry Dwight Chapin: Diseases of infants and children, New York, W. Wood and Co., 1911, p. 58 .

72 BUDIN, Pierre, The Nursling: the feeding and hygiene of premature \& full-term infants, London, Caxton Publishing Company, 1907, p. 165

73 Ibid.
} 
Unos años más tarde, el médico inglés Archibald Garrod presentaba de nuevo el amor y la empatía como condición indispensable para que se produjese de manera satisfactoria la práctica médica, exaltando de esta manera las cualidades que debía tener el médico dedicado a este periodo de la vida. ${ }^{74} \mathrm{Si}$ bien la narrativa de Garrod puede entenderse como una continuación del discurso que Charles West y otros pediatras generasen cincuenta años atrás, alrededor de estas fechas la visión mecanicista del niño empieza a permear la práctica pediátrica, que en aquel momento ya contaba con el reconocimiento de la profesión médica. ${ }^{75} \mathrm{Si}$ bien en tiempos de West la ausencia de una historia directa del paciente y la consiguiente insistencia en la importancia primordial del examen físico por parte del médico llevó a la comparación de la figura del pediatra con la del explorador, en estas fechas la práctica de la medicina infantil empezó a compararse con la práctica veterinaria. ${ }^{76} \mathrm{El}$ pediatra escocés Robert Hutchison empleó la analogía para descartar cualquier intento de construir una historia:

Los así llamados "síntomas subjetivos" de los niños no nos suponen ningún problema, porque no existen. La pediatría es en este aspecto como la veterinaria, el paciente es incapaz de dar cuenta de su sufrimiento y así te ves devuelto por entero a tu propia observación, que, por supuesto, hace que la necesidad de una examinación cuidadosa sea aún mayor. ${ }^{77}$

Unos años más tarde, Edmund Cautley recomendaba no mirar a los niños directamente a la hora de realizar un diagnóstico, ya que a estos "como a los animales, les desagrada intensamente que se les mire a los ojos". ${ }^{78} \mathrm{Al}$ igual que Robert Hutchison, Cautley comparaba las enfermedades de los niños con las de los animales, "porque en cada caso dependemos de las observaciones de los testigos y del examen físico, mientras que los síntomas ni nos ayudan ni nos engañan." ${ }^{79} \mathrm{Si}$, como sucedía con la metáfora del explorador, la analogía veterinaria reforzaba la necesidad de que el médico desarrollara una capacidad especializada para realizar una evaluación clínica directa del paciente infantil, a su vez conectaba con observaciones de finales del siglo XIX sobre las características animales de los niños de menor edad, que se consideraban una "recapitulación" de la evolución de las especies. En su introducción a Child Study, el pediatra escocés William Drummond detallaba las diversas maneras en que la ciencia había centrado su atención en la infancia. Mientras que el interés del filólogo viene de la esperanza de obtener una mejor comprensión del origen del lenguaje humano, el arqueólogo encuentra un valioso material de investigación en los dibujos infantiles. El antropólogo, en cambio, incapaz de encontrar un espécimen vivo del

74 GARROD, Archibald, Diseases of children; by various authors, BATTEN, Frederick, GARROD, Archibald y THURSFIEL, Hugh, (eds.), London, Edward Arnold, 1913, p.1.

75 Institucionalización que cristalizó en 1927 con la creación en Gran Bretaña de un cuerpo profesional de especialistas en salud infantil formalmente constituido: British Paediatric Association en 1927, a la que le seguiría, dos años más tarde, la fundación de la American Academy of Pediatrics.

76 GILLIS, Jonathan: "Taking a medical history in childhood illness: representations of parents in pediatric texts since 1850”, Bull Hist, 79, 393-429, (2005), p. 429.

77 HUTCHISON, Robert: Lectures on diseases of children, London, Edward Arnold, 1904, p. 4-5.

78 CAUTLEY, Edmund: The diseases of infants and children, New York, Paul B. Hoeber, 1912, p. 3.

79 CAUTLEY, Edmund: The diseases of infants..., p. 4. 
hombre primitivo, se vuelve hacia el niño como su representante más cercano. ${ }^{80} \mathrm{Si}$ bien unas líneas más abajo Drummond contrapone la figura del pediatra a la de estos profesionales, definiendo al médico de niños como un ser empático que entiende esta etapa de la vida como un momento único, su texto contiene influencias de la visión mecanicista de la infancia. Al igual que para Shinn y Hall, para este pediatra los movimientos expresivos de los neonatos inicialmente indican tan solo placer o dolor; así "sonrisas, risas y gorjeos", acompañados por movimientos de las extremidades, son señales tempranas de placer, mientras que la contracción hacia debajo de las esquinas de la boca y el fruncimiento del ceño señalan la aparición de lágrimas. ${ }^{81} \mathrm{~A}$ pesar de que Drummond reconocía la capacidad del niño de experimentar dolor, se remitía a la investigación de Preyer, introduciendo estas sensaciones en la sección dedicada a "movimientos reflejos". Su referencia a Preyer resulta especialmente relevante a la hora de hablar del primer llanto del niño, coincidiendo con el fisiólogo inglés en que este tiene un origen reflejo, "una visión mucho más razonable que la de Kant cuando habla de él como de un llanto de indignación e ira", o de la noción común, según la cual se debe al "dolor". ${ }^{82}$ El texto de Drummond pone de manifiesto el cambio en la concepción del niño, que se estaba fraguando en el marco de la pediatría como práctica institucionalizada. Alrededor de estas fechas, los pediatras comenzaron a aplicar la teoría del desarrollo infantil a sus prácticas médicas, equiparando el desarrollo del niño con el desarrollo animal, ${ }^{83}$ a la vez que introducían y establecían los estándares de normalidad biológica para estas etapas de la vida.

\section{Conclusiones}

El debate sobre el dolor infantil nos conduce a tres asuntos de gran peso en la historia de las ciencias y en la historia de la infancia. Por una parte, el problema de la falta de unidad científica ${ }^{84}$; en segundo lugar, el problema de las concepciones adultas de la infancia; por último, el problema de cómo se define, conceptualiza y trata el dolor. Los textos científicos y pediátricos empleados en este artículo muestran la falta de unidad de la ciencia a finales del siglo XIX: al mismo tiempo que el método científico iba transformando al niño en un objeto científico mecanicista, con la consiguiente negación del dolor infantil, los pediatras veían en los niños enfermos a "niños sin infancia", siendo su papel, por lo tanto, no solamente el de restaurar la salud del niño, sino también los valores de inocencia y bondad propios de la concepción romántica de la infancia. La fisiología experimental, al igual que la psicología evolutiva y conductista, no se volcaron al estudio infantil porque les interesaran los niños, sino porque albergaban la esperanza de descubrir hechos que pudieran arrojar algo de luz

80 DRUMMOND, William: The child: his nature and nurture, London, Dent, 1902, p. 4.

81 Ibid., p. 76,

82 Ibid., p. 73

83 Véase BUCKMAN, S: "Babies and. monkeys", The Nineteenth Century, 36, 743, (1984); KIDD, W: "The prehensile power of the hands of the human infant", Lancet, 150, 1009-1010, (1897), p. 1010

84 Véase, BIAGOLI, Mario y GALISON, Peter: The disunity of science : boundaries, contexts, and power, Stanford, Stanford University Press, 1996. 
sobre sus propias disciplinas. El ascenso de las ciencias históricas y su alianza con las teorías de la recapitulación, dieron al niño una posición sin precedentes en el dominio del conocimiento: dejaba de ser un asunto secundario o superfluo para convertirse en una fuente de saber clave para buen número de disciplinas. Sin embargo, es sorprendente cómo, en el mismo momento en que la infancia alcanza un interés tan notable para la psicología, parece desaparecer junto con su dolor. Las detalladas anotaciones científicas sobre los primeros movimientos y expresiones del infante, relacionadas con la sensación y el dolor, no se debían a un interés en el propio niño, sino a su función como índice del desarrollo evolutivo. En todas estas narraciones, el niño es la clave para comprender la mente del adulto y la aparición de la conciencia de sí. Mientras tanto, los pediatras estaban interesados en estudiar a los niños por el propio bien de los mismos. En contraposición a científicos y psicólogos, que veían en el niño a un adulto en ciernes, los médicos infantiles subrayaban la gran distancia que mediaba entre los niños y su asimilación como meros hombres y mujeres en miniatura. Así se explica que los derechos de los niños en el mundo pediátrico no se basaran en el derecho a ser tratados como futuros adultos, sino en la aislación del derecho a ser niño, a existir en un espacio segregado del mundo adulto. La reelaboración del ideal romántico de la infancia como tiempo que debe ser atesorado y protegido de la vida adulta, se vio complementado por un nuevo modelo médico. Aun así, no podemos olvidar que los niños sanos eran considerados un activo nacional y su salud, la salud del país.

Sin embargo, el debate sobre el dolor infantil da más información sobre la dominación cultural de ciertos ideales de la infancia que sobre la falta de unidad de las ciencias. Los debates provenientes del campo de la pediatría, la fisiología, la psicología evolutiva y conductual, recuperan los dos enfoques que, desde Locke y Rousseau, componían la visión occidental de la infancia. Por una parte, la infancia se considera un camino hacia la edad adulta, una fase fundamental en el desarrollo de un ser humano completo. Por otra, se respeta la infancia por ser una época de inocencia y de felicidad que debe ser preservada. La ambivalencia entre la infancia como refugio de la identidad personal o como testimonio de las civilizaciones pasadas, experimentó en el siglo XIX fuertes tensiones que, al analizar el problema del dolor infantil, se pueden detectar en nuestros actuales marcos de referencia. La investigación sobre la historia de la medicina y de las ciencias es un lugar adecuado para comprobar como la atención se desplaza a aspectos cada vez más concretos. La prueba se tiene en la correlación entre la atención social al niño, los avances y la especialidad de pediatría, que surge como tal cuando esa atención se formaliza e institucionaliza, en ese sentido de práctica social de investigación. El dolor en la infancia es un status sociológico, y una realidad médica y quirúrgica, que ha hecho surgir una ciencia especifica social e histórica y que puede servir de base a la evolución histórica de la visión del niño.

En lo que al propio dolor se refiere, el problema comienza con el desacuerdo sobre qué es el dolor. Recordemos que, mientras a finales del siglo XIX, Darwin y Preyer describían el dolor como un acto reflejo y aun así confirmaban la experiencia del dolor del infante basándose en sus observaciones, a medida que el conductismo se fue afianzando entre los psicólogos, los investigadores llegaron a la conclusión de que incluso una respuesta clara de desagrado era irrelevante para la cuestión de si los 
niños experimentaban dolor, ya que no era más que un reflejo, una respuesta automática a los estímulos. Ya bien entrado el siglo XX, la tan mencionada definición de la Association for the Study of Pain (IASP) definió el dolor como "una experiencia sensorial y emocional desagradable asociada con el daño potencial o efectivo de tejido, o descrito en términos de dicho daño" y afirmaba que "el dolor siempre es subjetivo, cada persona aprende a utilizar la palabra mediante experiencias relacionadas con lesiones tempranas". ${ }^{85} \mathrm{Si}$ bien se han exaltado las virtudes de este modelo, que entendía el dolor como un fenómeno subjetivo complejo, porque permitieron la visibilización de pacientes cuyo dolor había sido invisible durante tanto tiempo, como aquellos que padecían dolor crónico o terminal, también codificaron un prejuicio hacia las experiencias no verbales del dolor ${ }^{86}$ Por medio de su interpretación, la definición de la IASP impide que sean clasificadas como dolorosas las experiencias de individuos que no sean capaces de expresarse a sí mismos, ya que la autoexpresión se considera necesaria para atribuir el dolor. Pese a que la IASP enmendó su definición en 2003, añadiendo una nota adicional importante para el dolor infantil ("la incapacidad de comunicación verbal no niega la posibilidad de que una persona esté sufriendo dolor y necesite un tratamiento adecuado para aliviar el dolor"), Harold Merskey, miembro del comité interdisciplinario que propuso la primera definición de dolor, seguía defendiendo en 1996 que "solo podemos saber si alguien tiene dolor si nos indica de forma fehaciente que el dolor existe" ${ }^{87}$ Teniendo en cuenta que la cirugía y otros procedimientos invasivos se siguen practicando en infantes y niños debido a la creencia de que los neonatos son insensibles al dolor ${ }^{88}$ el problema del dolor en la infancia pone de manifiesto la necesidad de cuestionar nuestras nociones acerca del dolor y su tratamiento, al igual que las relativas al periodo que denominamos infancia.

\section{Referencias Bibliográficas}

ANAND, K. Y CRAIG, K: "New Perspectives on the Definition of Pain", Pain, 67, (1996), p.3-6.

BLANTON, Margaret Gray: "The behavior of the human infant in the first 30 days of life", Psychol Rev, 24, (1917), p. 456-483.

BIAGOLI, Mario y GALISON, Peter: The disunity of science : boundaries, contexts, and power, Stanford, Stanford University Press, 1996.

BOURKE, Joanna: The Story of Pain: From Prayers to Painkillers, Oxford, Oxford University Press, 2014.

85 La primera definición es de 1979, Pain, 6, 3, (1979), pp. 249-252.

86 ANAND, K. Y CRAIG, K: "New Perspectives on the Definition of Pain", Pain 67, (1996), p.3-6.

87 MERSKEY, Harold: "Response to Editorial: New Perspectives on the Definition of Pain", Pain, 66, (1996), p.209. Ya en 1970, Merskey había sugerido que la percepción del dolor por parte de los niños era inmadura y que esta habilidad se adquiría con la edad.

88 MIDMER, Deana: "Pain Perception in the Neonate: A Matter of Opinion", CAN.FAM.PHYSICIAN, 35 (1989), p. 1049-1054. 
BUDIN, Pierre, The Nursling: the feeding and hygiene of premature \& full-term infants, London, Caxton Publishing Company, 1907.

BUCKMAN, S: "Babies and monkeys", The Nineteenth Century, 36, 743, (1984); KIDD, W: "The prehensile power of the hands of the human infant", Lancet, 150, (1897), p. 1009-1010.

CARMICHAEL, James: Disease in children: a manual for students and practitioners, Edinburgh, Pentland, 1892.

CASTAÑEDA, Claudia: Figurations: Child, Bodies, Worlds, Durham, Duke University Press, 2002.

CAUTLEY, Edmund: The diseases of infants and children, New York, Paul B. Hoeber, 1912, p. 3.

CHAMBERLAIN, Alexander Francis: The child: a study in the evolution of man, London, W. Scott Ltd., 1900.

CHAMBERLAIN, David: "Babies don't feel pain: A century of denial in medicine", J Prenat Perinat Psychol Health, 14, (1999), 145-168.

CHAPIN, Henry: Diseases of infants and children, New York, W. Wood and Co., 1911.

COLON A. R. Colón y COLON P. A., Nurturing Children: A History of Pediatrics, Westport, Conn, Greenwood Press, 1999.

COOTER, Roger: "Introduction", en Roger Cooter (ed.):In the Name of the Child: Health and Welfare, 1880-1940, London, Routledge, 1992.

DAUNTON, M. J.: "Health and housing in Victorian London", Medical History, Supplement 11, (1991), p. 126-144.

DARWIN, Charles: The Expression of Emotions in Man and Animals, London, John Murray, 1872.

DARWIN, Charles: “A Biographical Sketch of an Infant”, Mind, 2, (1877), p. 285294.

DRUMMOND, William: The child: his nature and nurture, London, Dent, 1902.

FORSYTH, David: Children in health and disease: a study of child-life, London, John Murray, 1909.

ECKARDT, Georg, "Contributions to a History of Developmental Psychology: Internacional William T. Preyer Symposium”, Berlin and New York, Mouton, 1985.

EVANSON, Richard Tonson: A practical treatise on the management and diseases of children, Dublin: Fannin \& Co, London: Renshaw, and Longman, 1842.

GARROD, Archibald, Diseases of children; by various authors, BATTEN, Frederick, GARROD, Archibald y THURSFIEL, Hugh, (eds.), London, Edward Arnold, 1913.

GARRISON, Fielding y ABT, Arthur: Abt-Garrison History of Paediatrics, with New Chapters on the History of Paediatrics in Recent Times, Philadelphia and London, W.B. Saunders, 1965. 
GENZMER, Alfred: Untersuchugen Ueber die Sinneswahrhehumugen des Neugeborenen Menschen, Inaugural Dissertation, Halle, DE, Plötzche Buchhandlung, 1873.

GESELL, Arnold: Infant behavior: Its genesis and growth, New York and London, McGraw-Hill Book Company, 1934.

GILLIS, Jonathan: "Taking a medical history in childhood illness: representations of parents in pediatric texts since 1850", Bull Hist, 79, (2005), p. 393-429.

GILLIS, Jonathan, y Loughlan, Patricia: "Not just small adults: the metaphors of paediatrics", Arch Dis Child, 92, 11 (2007), pp. 946-947.

HALL, Stanley: Aspects of child life and education, New York y London, D. Appleton and company, 1921.

HESS, A: "On the necessity of practical instruction in the treatment of diseases of children", Lancet, 10 (1849), p. 341-342.

HOLT, Luther: The diseases of infancy and childhood: for the use of students and practitioners of medicine, New York: Appleton, 1987.

HUTCHISON, Robert: Lectures on diseases of children, London, Edward Arnold, 1904.

International Association for the Study of Pain (1979) "Pain Terms: A List with Definitions and Notes on Usage", Pain 6, p.249-252.

KIDD, W: "The prehensile power of the hands of the human infant", Lancet, 150, 1009-1010, (1897), p. 1010

KUSSMAUL, Adolf: Untersuchungen uber das Seelenleben des Neugebormen Menschem. Leipzig, DE, Winter, 1859.

LESCH, John: Science and Medicine in France. The Emergence of Experimental Physiology, 1790-1855, Cambridge, Mass. y Londres, Harvard University Press, 1984.

LOMAX, Elizabeth: "Small and Special: The Development of Hospitals for Children in Victorian Britain", Medical History, 16, London, Wellcome Institute for the History of Medicine, (1996), p. 383-400.

LORCH, Marjorie y HELLAL, Paula: "Darwin's "Natural Science of Babies"”, Journal of the history of the neurosciences, 19, 2, (2010), p. 140-157.

MAATER, Florence: Child behavior; a critical and experimental study of young children by the method of conditioned reflexes, Boston, The Gorham Press, 1918.

MEIGS, John Forsyth: A Practical Treatise on the Diseases of Children, Philadelphia, Lindsay and Blackiston, 1853.

MELZACK, Ronald y WALL, Patrick: "Pain Mechanisms: A New Theory", Science, 150, 3699, 1965, p. 971-9

MIDMER, Deana: "Pain Perception in the Neonate: A Matter of Opinion", CAN. FAM.PHYSICIAN, 35 (1989), p. 1049-1054.

MOSCOSO, Javier: Historia Cultural del Dolor, Madrid, Taurus, 2011. 
PREYER, William: The Mind of the Child: The development of the intellect, New York, NY, Arno Press, 1889.

PRODGER, Philip: Darwin's Camera, Oxford, Oxford University Press, 2009.

REY, Roselyne: The History of Pain, Cambridge, MA, 1995.

RODKEY, Elissa y PILLAI RIDDELL, Rebecca: "The Infancy of Infant Pain Research: The Experimental Origins of Infant Pain Denial", The Journal of Pain, 14, (2013), p. 338-350.

ROTCH, Thomas: "Iconoclasm and original thought in the study of pediatrics", Arch Pediatr, 7, (1891), p. 806-811.

STANLEY, Peter: For Fear of Pain: British Surgery, 1790-1850, Amsterdam-New York, Editions Rodopi, 2003.

STARR, Louis and WESTCOOTE, T. S, (eds), An American text-book of the diseases of children, Philadelphia, PA: Saunders, 1985.

SMITH, Jonathan: Charles Darwin and Victorian Culture, Cambridge: Cambridge University Press, 2006.

SHERMAN, Mandel y SHERMAN, Irene: "Sensori-motor Responses in Infants", Journal of Comparative Psychology, 5 (1925), p. 53-68;

SHERMAN, Mandel y SHERMAN, Irene: "Infant behavior", Comp Psychol Mono$g r, 12,(1936)$, p. 1-107

SHINN, Milicent: The biography of a baby, Boston and New York, Houghton Mifflin, 1900.

SHUTTLEWORTH, Sally: The Mind of the Child: Child Development in Literature, Science, and Medicine, 1840-1900, Oxford, Oxford University Press, 2010.

TANNER, Andrea: "Too many mothers? Female roles in Metropolitan Victorian children's hospital", in HENDERSON, John y PASTORE, Alessandro (eds.), The Impact of Hospitals, Oxford and Bern, Peter Lang, 2007.

TANNER, Andrea: "Choice and the children's hospital: Great Ormond Street Hospital patients and their families, 1855-1900', en BORSAY, A y SHAPELY, P (eds.), Medicine, Charity and Mutual Aid: The Consumption of Health and Welfare in Britain, c.1550- 1950, Aldershot, 2007.

VINCENT, Ralph: The nutrition of the infant, 4th ed, London, Bailliere, Tindall and Cox, 1913.

WEST, Charles, Lectures on the Diseases of Infancy and Childhood, London, Longman, Brown, Green \& Longmans, 1852.

ZELIZER, Viviana: Pricing the Priceless Child: The Changing Social Value of Children, New York, Basic Books, I985. 